\title{
AÇÃO ANTIFÚNGICA DE UM JATO DE PLASMA NÃO-TERMICO DE HÉLIO/AR COMPRIMIDO SOBRE BIOFILMES DE CANDIDA ALBICANS
}

\author{
Fernanda Ramos Figueira ${ }^{1}$ \\ Guilherme Redi Torello² \\ André Luiz S. Oliveira ${ }^{3}$ \\ Jhonatan S. Brandão de Lima ${ }^{4}$ \\ José Augusto Nunes Figueira ${ }^{5}$ \\ Homero Santiago Maciel ${ }^{6}$ \\ Rodrigo Savio Pessoa ${ }^{7}$ \\ Anelise Cristina Osório Cesar Doria ${ }^{8}$ \\ Sônia Khouri ${ }^{9}$
}

Resumo: Os biofilmes fúngicos são uma predominante causa de infecções crônicas associadas à utilização de cateteres e próteses, conferindo resistência aos antibióticos e fatores imunológicos do hospedeiro, sendo as leveduras, do gênero Candida spp, as mais frequentemente isoladas. O plasma não-térmico, operado à pressão atmosférica, vem ganhando destaque como uma nova estratégia antimicrobiana, inclusive para erradicação de biofilmes. Este trabalho teve como objetivo avaliar a eficácia da inativação de biofilmes de Candida albicans ATCC sobre substrato de poliuretano, utilizando jatos de plasma atmosférico de $6 \mathrm{~L} / \mathrm{min}$ de hélio e $4 \mathrm{~L} / \mathrm{min}$ de ar comprimido, alternando entre sistema contínuo e pulsado, com frequência de $60 \mathrm{~Hz}$ e distâncias de 10 a 30mm entre o bocal e substrato. Após tratamento, realizou-se a contagem das unidades formadoras de colônia e a análise morfológica da superfície do biofilme por Microscopia Eletrônica de Varredura. O melhor grupo foi o plasma de modo pulsado com distância de 30mm com redução de $92 \%$ das unidades formadoras de colônia, demonstrando ser uma tecnologia promissora para o controle de biofilmes de C. albicans.

Palavras-chave: Candida albicans; Biofilme; Plasma atmosférico; Modo contínuo; Modo pulsado.

\footnotetext{
${ }^{1}$ Graduanda em Biomedicina, Engenharia Elétrica e Engenharia Aeronáutica/Universidade do Vale do Paraíba, Brasil. E-mail: ferfig510@gmail.com.

2 Graduando em Biomedicina, Engenharia Elétrica e Engenharia Aeronáutica/Universidade do Vale do Paraíba, Brasil. E-mail: gui_torello@hotmail.com.

${ }^{3}$ Graduando em Biomedicina, Engenharia Elétrica e Engenharia Aeronáutica/Universidade do Vale do Paraíba, Brasil. E-mail: aluizsoliveira@gmail.com.

${ }^{4}$ Graduando em Biomedicina, Engenharia Elétrica e Engenharia Aeronáutica/Universidade do Vale do Paraíba, Brasil. E-mail: jhonatan_Ima@hotmail.com.

5 Doutorando em Engenharia Aeronáutica/Instituto Tecnológico de Aeronáutica - Divisão de Engenharia Mecânica, Brasil. E-mail: jose.augusto.figueira@gmail.com.

${ }^{6}$ Docente da Univap e Doutor em Electrical Discharges And Plasmas/ University of Oxford - Inglaterra, Brasil. Email: odairtur@gmail.com.

7 Docente da Univap e Doutor em Ciências na Física de Plasmas/ Instituto Tecnológico de Aeronáutica, Brasil. E-mail: rspessoa@univap.br.

8 Doutoranda em Engenharia Biomédica/ Universidade do Vale do Paraíba, Brasil. E-mail: ane.doria@gmail.com.

${ }^{9}$ Docente da Univap e Doutora em Microbiologia/ Universidade de São Paulo, Brasil. E-mail: soniak@univap.br.
} 